\title{
Cutaneous vascular \& sudomotor responses to heat-stress in smokers \& non-smokers
}

\author{
Nicole E Moyen, Hannah A Anderson, Jenna M Burchfield, Matthew A Tucker, Melina A Gonzalez, \\ Forrest B Robinson, Matthew S Ganio* \\ From 15th International Conference on Environmental Ergonomics (ICEE XV) \\ Portsmouth, UK. 28 June - 3 July 2015
}

\section{Introduction}

As approximately one billion people worldwide are chronic smokers [1] it is important to determine smokers' thermoregulatory responses to heat-stress. Although local maximal vasodilation may be attenuated in smokers [2], skin blood flow responses during whole-body heat stress are unknown. Moreover, it is unknown if sweat rate is altered in smokers; theoretically the binding of nicotine to nicotinic acetylcholine receptors [2] may initiate an earlier onset of sweating during whole-body heat stress compared to non-smokers [3]. The purpose of this study was to compare cutaneous vascular and sudomotor responses to whole-body passive heat-stress between smokers and non-smokers.

\section{Methods}

Nine male chronic smokers [SMK; 10 (6) cigarettes/day for $11.8(9.5) \mathrm{y} ; 26(8) \mathrm{y} ; 177.7(6.6) \mathrm{cm} ; 80.6 \pm 21.1 \mathrm{~kg}]$ and 13 male non-smokers [N-SMK; 28 (9) y; 177.6 (6.8) $\mathrm{cm} ; 77.2(8.2) \mathrm{kg}$ ] were matched for age, height, body mass, and exercise habits (all $\mathrm{p}>0.05$ ). Subjects were passively heated via water-perfused suits until gastrointestinal temperature $\left(\mathrm{T}_{\mathrm{gi}}\right)$ increased $1.5^{\circ} \mathrm{C}$. Local sweat rate (LSR) via ventilated capsule and cutaneous vasomotor activity (CVC) via Laser Doppler on the forearm were continuously recorded; blood pressure, heart rate, sweat gland activation (SGA), sweat gland output (SGO), $\mathrm{T}_{\mathrm{gi}}$, and mean-weighted skin temperature $\left(\mathrm{T}_{\text {sk }}\right)$ were taken at baseline and each $0.5{ }^{\circ} \mathrm{C} \mathrm{T}_{\mathrm{gi}}$ increase. LSR and CVC onsets and sensitivities were calculated with mean body temperature $\left(\mathrm{T}_{\mathrm{b}}\right)=0.9 * \mathrm{~T}_{\mathrm{gi}}+0.1 * \mathrm{~T}_{\mathrm{sk}}[4]$.

\footnotetext{
* Correspondence: msganio@uark.edu

Human Performance Laboratory, Department of Health, Human Performance, and Recreation, University of Arkansas, Fayetteville, AR, USA
}

Table 1 Mean (SD) CVC and LSR parameters on the forearm for SMK and N-SMK during passive heat stress

\begin{tabular}{|c|c|c|c|}
\hline & Measurement & Smokers & $\begin{array}{l}\text { Non- } \\
\text { smokers }\end{array}$ \\
\hline \multirow[t]{3}{*}{ CVC } & CVC onset $\left(\Delta \mathrm{T}_{\mathrm{b}}\right.$ from baseline, $\left.{ }^{\circ} \mathrm{C}\right)$ & $\begin{array}{c}0.31 \\
(0.12)\end{array}$ & $0.61(0.21)^{*}$ \\
\hline & CVC plateau (\% of max) & $\begin{array}{c}68.4 \\
(27.4)\end{array}$ & $68.4(21.6)$ \\
\hline & CVC sensitivity $\left(\Delta \% \max\right.$ per $\left.{ }^{\circ} \mathrm{C} \Delta \mathrm{T}_{\mathrm{b}}\right)$ & $\begin{array}{l}82.5 \\
(46.2)\end{array}$ & $58.9(23.3)$ \\
\hline LSR & LSR onset $\left(\Delta \mathrm{T}_{\mathrm{b}}\right.$ from baseline, $\left.{ }^{\circ} \mathrm{C}\right)$ & $\begin{array}{c}0.35 \\
(0.14)\end{array}$ & $0.52(0.19)^{*}$ \\
\hline & $\mathrm{LSR}$ plateau $\left(\mathrm{mg} \cdot \mathrm{cm}^{-2} \cdot \mathrm{min}^{-1}\right)$ & $\begin{array}{c}0.79 \\
(0.26)\end{array}$ & $1.00(0.13)^{*}$ \\
\hline & $\begin{array}{l}\text { LSR sensitivity }\left(\Delta \mathrm{mg} \cdot \mathrm{cm}^{-2} \cdot \mathrm{min}^{-1}\right. \\
\left.\text { per }{ }^{\circ} \mathrm{C} \Delta \mathrm{T}_{\mathrm{b}}\right)\end{array}$ & $\begin{array}{c}0.60 \\
(0.40)\end{array}$ & $0.63(0.21)$ \\
\hline
\end{tabular}

*Significant difference between groups $(p<0.05)$.

\section{Results}

No differences existed between SMK and N-SMK for $\mathrm{T}_{\mathrm{gi}}, \mathrm{T}_{\mathrm{sk}}, \mathrm{T}_{\mathrm{b}}$, heart rate, mean arterial pressure, LSR, $\mathrm{CVC}$, and SGA with each $0.5{ }^{\circ} \mathrm{C} \mathrm{T}_{\mathrm{gi}}$ increase (all $\mathrm{p}>$ 0.05). Overall, SGO tended to be lower in SMK than $\mathrm{N}$-SMK $[\mathrm{SMK}=5.94(3.49)$ vs. $\mathrm{N}-\mathrm{SMK}=8.94(3.99)$ $\left.\mu \mathrm{g} \cdot \mathrm{gland}^{-1} \cdot \mathrm{min}^{-1} ; \mathrm{p}=0.08\right]$.

\section{Discussion}

Smokers' CVC and LSR onsets occurred at an earlier $\mathrm{T}_{\mathrm{b}}$ than non-smokers, possibly because heat stress enhances nicotine kinetics (i.e. binding of nicotine to nicotinic acetylcholine receptors; $[2,3]$ ). The lower LSR at plateau during whole-body heating might indicate a thermoregulatory impairment in young smokers, and is likely a result of decreased sweat gland output and not activation. 


\section{Conclusion}

Compared to non-smokers, smokers had an earlier onset but similar sensitivity (i.e. increase in response per increase in $\mathrm{T}_{\mathrm{b}}$ ) for sweating/cutaneous vasodilation. These data suggest that overall, most young chronic smokers' thermoregulatory responses to whole-body passive heat stress are not impaired.

Published: 14 September 2015

\section{References}

1. Alwan A: Global status report on noncommunicable diseases 2010. World Health Organization; 2011.

2. Kilaru S, Frangos SG, Chen AH, Gortler D, Dhadwal AK, Araim O, Sumpio BE: Nicotine: a review of its role in atherosclerosis. J Am Coll Surg 2001, 193(5):538-546.

3. Ogawa T: Local effect of skin temperature on threshold concentration of sudorific agents. J Appl Physiol 1970, 28:18-22.

4. Stolwijk JA: A mathematical model of physiological temperature regulation in man. National Aeronautics and Space Administration 1971.

doi:10.1186/2046-7648-4-S1-A98

Cite this article as: Moyen et al: Cutaneous vascular \& sudomotor responses to heat-stress in smokers \& non-smokers. Extreme Physiology \& Medicine 2015 4(Suppl 1):A98.

Submit your next manuscript to BioMed Central and take full advantage of:

- Convenient online submission

- Thorough peer review

- No space constraints or color figure charges

- Immediate publication on acceptance

- Inclusion in PubMed, CAS, Scopus and Google Scholar

- Research which is freely available for redistribution

Submit your manuscript at www.biomedcentral.com/submit 